\title{
Millimeter Wave Channel Estimation and Beam forming Techniques
}

\author{
Y. Srinivasa Rao, R.Madhu
}

\begin{abstract}
Millimeter-wave(mm-wave) frequencies offer higher bandwidth communication channels. Most of the wireless communication equipment operates with $6 G H Z$ carrier frequencies, where the millimeter-wave communication systems are operated in the frequency range of $30 \mathrm{GHZ}$ to $300 \mathrm{GHZ}$. Channel estimation will become a difficult task when the number of antennas is more than the number of radiofrequency chains. Millimeter-wave communication has received large attention from research fraternity and industry. In this paper, we listed the evolution and advancements in antenna beam forming, at the same time we outline some of the channel estimation techniques such as Auxiliary Beam pair (ABP), Adaptive algorithm based Codebook design (AABCD), Estimation of Signal Parameters by Rotational Invariance Technique (ESPRIT).
\end{abstract}

Keywords: AABCD, ABP, Beamforming, ESPRIT, mm-wave.

\section{INTRODUCTION}

A Millimeter-wave massive MIMO channel estimation and beamforming is an emerging new research field. To meet this challenging demand, the capacity enhancement of wireless links needs to be addressed. In this backdrop, millimeter wave technology for indoor and outdoor wireless communication has emerged as a new frontier to deploy high-speed data links. The feasibility of indoor implementation of millimeter-wave links has been successfully demonstrated by so many stakeholders.

To overcome the disadvantage of path loss in millimeter-wave the number of antennas placed at the Base Stations (BS) and Mobile Stations (MS) is increased but the number of RF chains is less compared to that of the number of antennas. A group of antennas is placed in an array for the transmission of one or more spatially directive signals. This technique is known as precoding. Precoding is used to increase spectral efficiency, in this technique same spatially directive signals are fed to each antenna in the array and the signals emitted by the antenna array are controlled by analog phase shifters. In order to reduce the power consumption and cost the precoding is implemented in both analog and digital domains .The benefit of pre-coding is that spectral efficiency is improved. The level of data that is transmittable over a certain network is spectral efficiency or bandwidth capacity on a specific communications system. Pre-coding hybrid is used since cost and energy consumption are reduced. It has both the digital and the analog section. The Channel State
Information (CSI) is required in both sections. It becomes harder for antennas to access the number of RF chains. Several inputs are therefore difficult to precisely measure multiple high-dimensional channels. The massive MIMO offers more benefits than MIMO points.

A multi-resolution codebook allows an adaptive canal approximation algorithm to build a beamforming vector with different values of its bandwidth. First of all, it is done for one way only and then multipath cases can be extended. In addition, quantified beam control vectors are considered other than the pre-coding vectors. The auxiliary beam-based pairing method is used to determine the angles of the signal sent and received by the antennas, which are defined by AOA and AOD. That auxiliary beam pair that evaluates the angles of the channel allows an amplitude comparison. The ESPRIT method can be utilized in practical cases as the selection process has many disadvantages. Eigenvalue decomposition (EVD) is done by means of a matrix that separates the signal and noise subspaces after which a different matrix which is the actual matrix is determined and EVd is again made, and therefore angles or directions of the signals can be measured. A simple ESPRIT approach involves taking covariant matrices of the received channel. IEEE 802.11ad The IEEE 802.11ad protocol for beamforming in three phases is specified in a selectional protocol. (1) Sector Level Sweep Phase: SLS is a gross system in which antennas use quasiOmni patterns to select the best transmit and receive antenna sector optionally. SLS phase is a gross process. (2) Beam Refining Phase (BRP): The beam refinement phase trains the beam and receives antenna arrays to pick a beam pattern pair with fine beam widths after the best sector pair has been established. 3) BT Phase: The optional beam tracking phase is used to adjust for channel changes during data transmission. Beam tracking is done by the TRN fields for data packets. Beam tracking is carried out. It also includes the automatic gain control (AGC) field in which a series of beam directions are changed to calculate a suitable AGC gain sequentially. The field TRN was designed to improve the precise tap delay estimate for each Channel Estimate (CE) beam. 


\section{System model:}
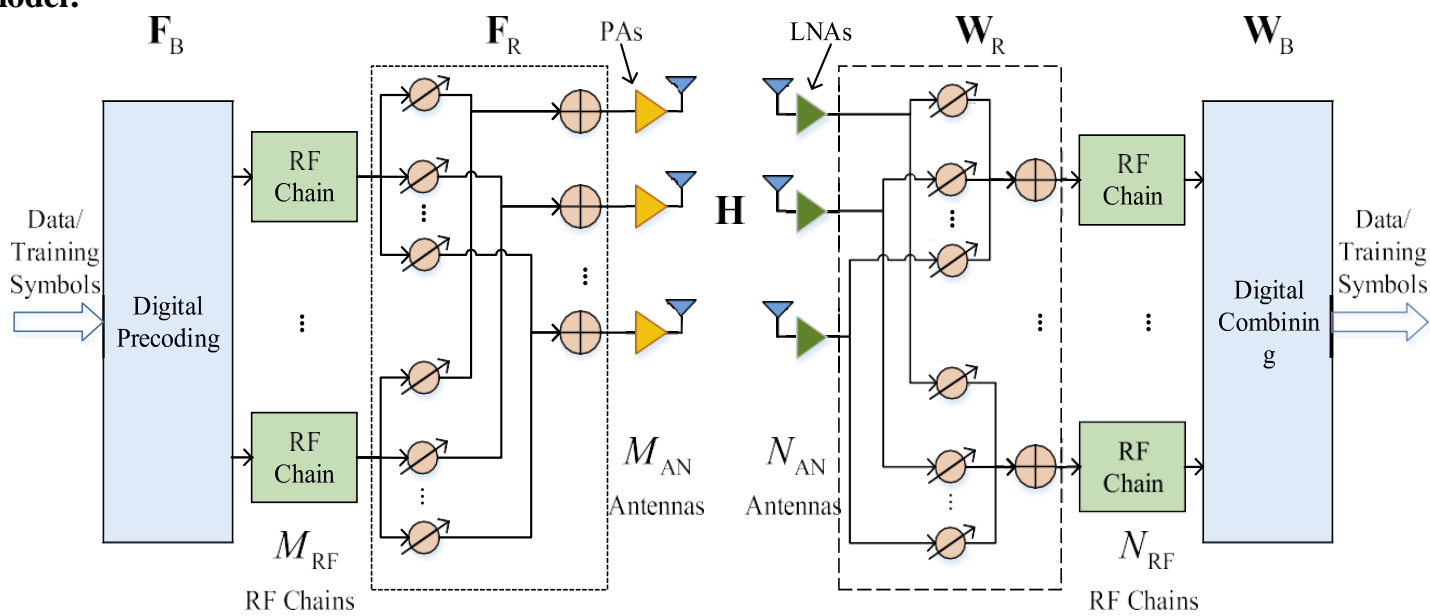

Table 1: Outdoor Millimeter Summary of Study on Campaigns

\begin{tabular}{|c|c|c|c|c|c|}
\hline $\begin{array}{c}\text { Used } \\
\text { carrier } \\
\text { frequency }\end{array}$ & $\begin{array}{l}\text { Investigation } \\
\text { Location }\end{array}$ & Range of RF Bandwidth & $\begin{array}{l}\text { Maximum } \\
\text { Tx/Rx } \\
\text { Length }\end{array}$ & $\begin{array}{r}\text { Evaluated } \\
\text { Parameters }\end{array}$ & $\begin{array}{r}\text { Application } \\
\text { for the target }\end{array}$ \\
\hline \multirow{5}{*}{$28.0 \mathrm{GHz}$} & metropolitan & $\begin{array}{c}5.0 \mathrm{KHz} \\
-1.0 \mathrm{GHz} @ 28-30 \mathrm{GHZ}\end{array}$ & $1.50 \mathrm{Km}$ & Path-Loss & $\begin{array}{l}\text { Point-to-poin } \\
\mathrm{t}\end{array}$ \\
\hline & $\begin{array}{l}\text { Garden(Green } \\
\text { House) }\end{array}$ & $5.0 \mathrm{KHz}$ & $0.90 \mathrm{Km}$ & $\begin{array}{l}\text { Depolarization of } \\
\text { Signal, Loss of } \\
\text { Foliage }\end{array}$ & LMDS \\
\hline & Sub metropolitan & $18.0 \mathrm{MHz}$ & $2-6 \mathrm{Km}$ & Path-Loss & LMDS \\
\hline & $\begin{array}{l}\text { Impenetrable } \\
\text { street }\end{array}$ & $800.0 \mathrm{MHz}$ & $\begin{array}{l}200 \mathrm{~m}-500 \\
\mathrm{~m}\end{array}$ & $\begin{array}{c}\text { AoD, Statistics of } \\
\text { AoA/ } \\
\text { outage,Path-Loss of } \\
\text { Omni directional } \\
\text { model }\end{array}$ & $\begin{array}{l}\text { Cellular/ } \\
\text { Point-to- } \\
\text { point }\end{array}$ \\
\hline & rooftop & NA & $40.0 \mathrm{~m}$ & $\begin{array}{l}\text { Matrix condition } \\
\text { number of MIMO } \\
\text { channel } \\
\end{array}$ & mobile \\
\hline $38.0 \mathrm{GHz}$ & $\begin{array}{l}\text { Metropolitan/ } \\
\text { Moderately } \\
\text { Impenetrable } \\
\text { street }\end{array}$ & $\begin{array}{l}800.0 \mathrm{MHz} \\
800.0 \mathrm{MHz}\end{array}$ & $\begin{array}{l}200.0-930 . \\
0 \mathrm{~m}\end{array}$ & $\begin{array}{c}\text { Delay spread of } \\
\text { RMS, Path-Loss } \\
\text {,Statistics of Outage }\end{array}$ & $\begin{array}{l}\text { mobile } \\
\text { mobile }\end{array}$ \\
\hline $\begin{array}{l}55.0-60.0 \mathrm{G} \\
\mathrm{Hz}\end{array}$ & $\begin{array}{l}\text { Metropolitan/ } \\
\text { Moderately } \\
\text { Impenetrable } \\
\text { street }\end{array}$ & $\begin{array}{c}\text { N.B.FM/200.0MHz-5.0G } \\
\mathrm{Hz}\end{array}$ & $23.0-400 \mathrm{~m}$ & $\begin{array}{c}\text { Statistics of } \\
\text { AoA,mean,path-loss, } \\
\text { envelop of } \\
\text { fading,Distribution } \\
\text { of AoA, Delay } \\
\text { spread of RMS }\end{array}$ & $\begin{array}{c}\text { Mobile } \\
\text { Radio/MM } \\
\text { wave mobile }\end{array}$ \\
\hline 73.0GHz & $\begin{array}{l}\text { Metropolitan(dor } \\
\text { m) }\end{array}$ & $800.0 \mathrm{MHz}$ & $200.0 \mathrm{~m}$ & PDP, Path-Loss & $\begin{array}{l}\text { Mobile/Cellu } \\
\text { lar }\end{array}$ \\
\hline $\begin{array}{l}81.0-86.0 \mathrm{G} \\
\mathrm{Hz}\end{array}$ & $\begin{array}{l}\text { Metropolitan(stre } \\
\text { et gorge) }\end{array}$ & $800.0 \mathrm{MHz}-5 \mathrm{GHz}$ & $\begin{array}{l}200.0-685 . \\
0 \mathrm{~m}\end{array}$ & $\begin{array}{c}\text { Delay spread of } \\
\text { RMS, Path-loss,PDP }\end{array}$ & $\begin{array}{l}\text { Radio of } \\
\text { E-Band }\end{array}$ \\
\hline
\end{tabular}




\section{FORMING MULTI USER COMPETITOR BEAM}

The existing wave communication standards for indoor millimeters plan a single transmission per time-space. The high loss of propagation in millimeter-wave connections can be exploited to create multiple communication links to directions that can simultaneously function to increase network capacity. However, it is necessary to address the problem of mutual interference caused by the concomitant connections in a short coverage area, which can have a major impact on concomitant production. The solution lies in the design of efficient protocols for MAC / cross-layer and transmission schedules for spatially multiplexed concurrent connections. The simultaneous beams for several simultaneous links have to take mutual interference into account in determining the best transmission/reception beam for each connection. In order to select beams for these competing connections, the maximum rates and not individual rates are optimized. The sub-optimal solution can be sought via the maximization of the SINR obtained for two or three of the connections. For the achievement of the highest MAC throughput, it is necessary to have efficient multi-user transmission schedules for millimeter-wave communication. The question of surfaced ness due to the narrow directional beam is particularly important. In the presence of several links for different antenna configurations (Omnidirectional and directions), analyzed for SINR in a millimeter WPAN system is performed in. A first-come-first-serve scheduling approach is being introduced by the researchers using beamforming knowledge to measure SINR. The multiple-path schedules multi-hop (MPMH) based on recent work on the hybrid beamforming spatial multiplexing are shown that the optimal path choice, traffic distribution, and transmission schedulation increases the space reuse on a millimeter-wave WPAN. The beam selection and transmission schedule joint consideration are dealt with in the text on which the writers submitted. Useful insights for optimal beam width selection and number of scheduled concurrent connections. The multi-user concept can be expanded to assess its performance with $5 \mathrm{G}$ outdoor millimeter-wave systems and cellular backhaul connections. For the future, it will provide crucial inputs for dense HetNets development and deployment.

\section{JOINT TRANSMIT- RECEIVE BEAM FORMING}

Setting optimum weight vectors for an antenna transmitting and receiving ends will result in a high scale gain to optimize received SNR / SINR before signal transmission. If CSI is available at both ends, the optimum vectors of weight are determined by maximizing suitable performance criteria, like the SNR receiver. For the frequency selective channels with a large number of multipath, designing joint transmitter-receiver analog strap forming algorithms is difficult because of the restriction of scalar weight per antenna. Iterative joint algorithms take into account this limit and calculate the transmission and obtain weights to maximize SNR, which regards the energy in the delayed paths as an additional noise input to the equalizer. Nevertheless, this suboptimal method requires full knowledge of the pairs of MIMO channels channel pulse response (CIR) on both sides which transmit and receive enormous overheads and that are not suitable for high data rate systems.

The energy in the late paths to increase the average symbol energy in the Equaliser Input is used as a way to circumvent this problem. Consequently, only internal products between all transmitted CIR pairs are the CSI necessary for joint optimization. Therefore, in real-time, the amount of CSI to be measured depends only on the transmitted number and antennas obtained and not the dispersal time of the network. You can avoid the need to obtain CSI with the help of the beamforming technique based on the codebook. Another approach is to implement a more robust adaptive belt shaping. It, however, includes the development of new methods for joint beam formation that do not require prior CSI. In a multifunctional scenario, it should be examined in the LOS and NLOS conditions for millimeter-wave communications the performance of joint beam sharing techniques. To achieve this goal it could exploit the quasi-static and sparse nature of the millimeter-wave channel.

\section{FORMATION OF HYBRID BEAM}

Recent research has focused heavily on the development of hybrid beams, a low-complex and cost-effective solution for the use of large-scale waving communications. The section reviewed above discussed the best precoding approaches for hybrid beam design. The combined precoding and reception of joint maximum transmitter remains a huge task. A joint transmission is shown to perform a classical, digital beam formation-based Selection of antennas schemes as an average SNR gain in the selective $60 \mathrm{GHz}$ frequency channels. The directionalities generated by phased antenna arrays in RF beam pattern and the space heterogeneity offered by multiple baseband processing modules are used for a cost-effective time-domain hybrid beamforming system.

A reliable beamformer is designed to hold the SINR for every client with minimal maximum transmittance power to preserve the performance of the signal under unpredictable multiple access and intersymbol interference scenarios for wireless streaming. Nonetheless, the degree to which the SINR goal can be reached in phase change sensitively. The plan mitigates intervention and phase uncertainties jointly. An analogical beam configuration with array antennas and electronic pre-encodes with multiple RF strings incorporates a multi-beam transmission diversity scheme for single-use MIMO (SU-MIMO) with a single-stream transmission using a hybrid beamforming architecture. The method uses limited, quantitative feedback to get data about the favorite analog and digital pre-coders. At the receiver for practical CSI measures, the Baseband processing is carried out.Hybrid precode algorithms with low complexity build and energy-efficient hybrid beam

model architectures are among the research tasks for $5 \mathrm{G}$ 
Millimeter-wave systems. for large MIMO implementation, The guiding principles for the designs of these systems are the study of the number of optimal streams and the realized spectral efficiency. A multi-User MIMO beam architecture, with a multi-user multi-user link, based on a dual-polarized planar antenna array, which reduces hardware costs. The authors propose a regularly designed iterative algorithm for the basic belt and RF precoder layout, as well as a basic band equalizer, and evaluate the power allocation of multi-stream data within the frequency domain to optimize the minimum SINR. Codebook based beam learning with a sparse millimeter-wave estimate AoD / AoA is an interesting problem. An important problem to address is the design of effective training sequences to reduce computational complexity as well as to achieve energy efficiency.

\section{GREEN COMMUNICATION USING BEAM LEARNING}

While research into $5 \mathrm{G}$ communications in the next decade focuses on meeting the expected large demand for traffic to provide seamless ubiquitous networking, due attention must also be paid to developing energy-efficient systems at a minimum footprint. For LSAS-based solid MIMO technology, energy efficiency is of particular importance. Among several problems, the co-design aspect energy efficiency-spectral efficiency (EE-SE) is particularly important. The effort to further leverage the energy savings provided by the hybrid beamforming architecture has been made to establish, in the context of a variable number of RF chains and number of antenna elements, the optimum operating point, also known as the green spot in the EE-SE curve. The basic concept is to optimize EE for a given SE in the optimization of device parameters. In order to obtain optimum EE-SE output, the EE-SE relationship at the green point is tested, with a derivative corresponding to the maximum number of RF chains. Joint baseband RF for pre-coder design in multi-user situations with interference management for the low-complexity energy-efficient sub-connected hybrid beamforming architecture is an interesting challenge.

\section{Secure Communications Beam Forming}

Antenna Subset Modulation is a novel method by using a sub-set of antennas in the array to modulate an antenna radiation pattern at the symbolic rate. This leads to a directional radiation pattern that projects a clearly defined constellation in the desired direction in other directions and randomized constellation. This method utilizes the large-scale antenna array of millimeter-wave systems to implement an inherent and robust safety mechanism with eavesdroppers to produce a lowly complex directional modulation system. Analog beam shaping coding can be used in MMB systems to ensure safe downlinking communication in cellular networks. The secrecy throughput is significantly higher than the traditional cellular network using delay-tolerant and delay controlled base station transmission modes. In the delay-tolerant mode even, when large antenna arrays are being used, the upper limit to secrecy bandwidth is reached for delay-bound mode. The secrecy throughput is very high for broad millimeter-wave bandwidths. The beam connections form a useful mechanism in millimeter-wave communications to improve security and privacy. In order to exploit the benefits of beam formation, new schemes need to be further developed to include security for medium and long-range outdoor communication.

\section{BEAM FORMING COMPRESSIVE SENSING}

Compressed channel sensing provides a promising method of estimating the split multifaceted channels and is widely used to reduce the complexity of hybrid beamforming systems in signal processing. The traditional least-square estimator requires a pilot-sequence-length of $\mathrm{K}$ of $\mathrm{Nt}$, whereby $\mathrm{Nt}$ is the number of antenna transmission components, as compared with the pilot-sensing approach requiring a significantly lower $\mathrm{K}$ of the $\log (\mathrm{Nt})$ size. It decreases substantially the amount of overhead preamble. For channel acquisition and monitoring is also used in the compressive sensing method. By using several compressive training light bulbs to calculate the channel, and based on the same, the technique uses a compressive tracking algorithm to further reduce the number of beacons. The technology is simplified by hardware design with user mobility. Suitable compressed estimation method does not require channel input information for multipattern millimeters, and it uses multilevel beamforming code building to adjust estimates for arrival and departure angles of all sparse channel multi patterns. Results of simulation show that the precoding benefits from the proposed algorithms are comparable to those obtained by feedback channel systems.

In large millimeter wave MIMO systems the compressive sensing of a two-step process consisting of coarse or fine channel estimates is used. The design consists of SVD-based methods of beam construction. The system obtained good results up to a range of 10 meters under low SNR conditions. There has just been a promising compression sensing approach in the millimeter-wave uplink multi-user channel for a selection of the beam, without the need for a specific estimate of the channel. Compressed sensing is a fruitful area in signal processing, especially with the sparse, multipath channel characteristics of millimeters of wave communication. It should be a key technique to model hybrid pre-coding algorithms computer-efficient. In developing precise methods for channel estimation for adaptive transmission beamforming, compressed sensing can also be critical.

\section{CONCLUSION}

With its large available bandwidth in the mmWave frequency range and the huge capacity gains from the massive antenna array system, MMWave massive MIMO technology aims to take advantage of promising prospects. In this study,

we presented a summary of the principles and strategies for large MIMO systems of mmWave. This illustrates how new network technologies are unique as compared to previous structures, from which main research problems and directions are derived and described. Alongside new implementations, standardisation and business models, though research, 
demonstration and field trials for candidate technologies and prototypes are underway through various academic and industry initiatives, integrated device implementation and performance evaluation remains open issues. When we head into 2020, mmWave massive MIMO work will begin to evolve, and there will be new trends. While there is a lot of problems, mmWave's large MIMO offers great potential and prospects to carry out the 1000-fold cellular network capacity search. New frontiers in cellular services and applications will be established with technology.

Agorithms that are adaptive in nature are used by the design of a codebook that has a multi-resolution. Their quality is assessed with one direction. This approach provides better spectral efficiency and progress values because it is merely complex. The channel estimate provided by Auxiliary Beam Pair (ABP) provides high angle resolution. The amplitude correlation for the auxiliary beam pair can be achieved. The angles of arrival and departure can then be calculated by using the 2D ESPRIT algorithm. You can get the track gains using the Last Square Estimate (LSE) method. The computational complexity is minimized by SVD-based preconditioning.

\section{REFERENCES}

1. Heath R. W et all., “An overview of signal processing techniques for millimetre wave MIMO systems," IEEE J. Sel. Topics Signal Process., vol. 10, no. 3, pp. 436_453, Apr. 2016.

2. Kutty Shajahan et all., "Beamforming for Millimeter Wave Communications: An Inclusive Survey" IEEE Communications Surveys \& Tutorials, Vol. 18, No. 2, Second Quarter 2016.

3. Miao $\mathrm{H}$ et all., "2-D unitary ESPRIT based joint AOA and AOD estimation for MIMO system," in Proc. IEEE 17th Int. Symp. PIMRC, Sep. 2006.

4. Kalyani et all., " Survey on channel estimation techniques for mm wave massive MIMO with hybrid precoding" IOSRISSN (e): 2250-3021, ISSN (p): 2278-8719 Vol. 09, Issue 5 (May. 2019), PP $52-58$

5. Swindlehurst A. Lee, et all.,"Millimeter-Wave Massive MIMO:The Next Wireless Revolution?",IEEE Communications Magazine • September 2014

\section{AUTHORS PROFILE}

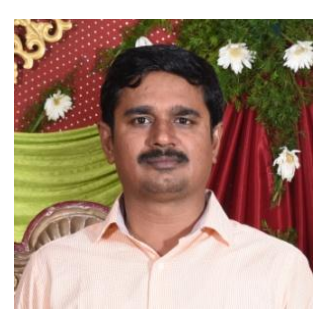

Madhu Ramarakula was born in Warangal district, INDIA in 1980. He received the B.E degree in Electronics \& Communication Engineering from Osmania University, Hyderabad, India, in 2003 and M.Tech degree in Communication Systems from Jawaharlal Nehru Technological University Hyderabad, India, in 2009 and Ph.D degree in Electronics \& Communication Engineering from Andhra University, Visakhapatnam, India, in 2014. $\mathrm{He}$ is presently working as an Assistant Professor in the Department of Electronics \& Communication Engineering, University College of Engineering Kakinada (A), JNTUK Kakinada, India. He has 10 years of teaching experience. He has published more than 30 research papers in various reputed national and international Journals and conferences. His research interests include Mobile communications, satellite communications and GPS. He is a member of IEEE. from JNTUK,Kakinada. His research interests are Mobile Communications. He has 5 publications in various national, international Journals.

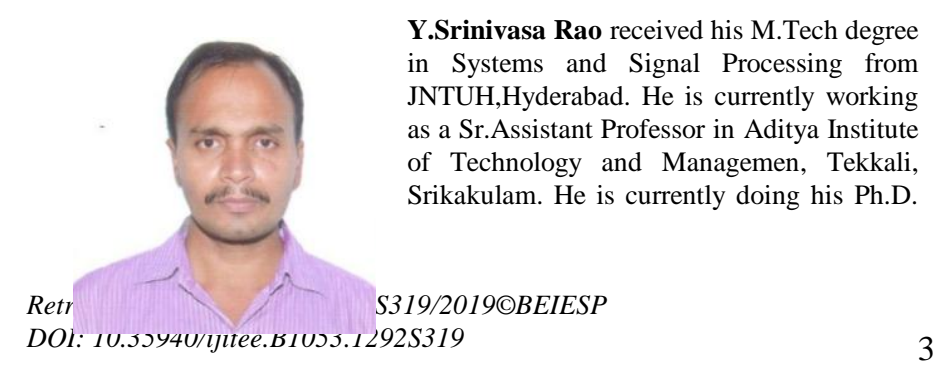
in Systems and Signal Processing from of Technology and Managemen, Tekkali, Srikakulam. He is currently doing his Ph.D. 